Research Article

\title{
Dynamic Response of an Inhomogeneous Elastic Pile in a Multilayered Saturated Soil to Transient Torsional Load
}

\author{
Kaifu Liu $\mathbb{D D}^{1}$ and Zhiqing Zhang $\mathbb{D}^{2}$ \\ ${ }^{1}$ School of Civil Engineering and Architecture, Zhejiang Sci-Tech University, Hangzhou 310018, China \\ ${ }^{2}$ School of Landscape Architecture, Zhejiang A\&F University, Hangzhou 311300, China \\ Correspondence should be addressed to Kaifu Liu; liukaifu@zstu.edu.cn
}

Received 2 February 2021; Revised 20 March 2021; Accepted 26 March 2021; Published 12 April 2021

Academic Editor: Zhongjin WANG

Copyright (c) 2021 Kaifu Liu and Zhiqing Zhang. This is an open access article distributed under the Creative Commons Attribution License, which permits unrestricted use, distribution, and reproduction in any medium, provided the original work is properly cited.

\begin{abstract}
In this paper, we solve the dynamic response of an inhomogeneous elastic pile embedded in a multilayered saturated soil and subjected to a transient torsional load via a semianalytical method. To portray the inhomogeneity of the pile and the stratification of surrounding soil, the pile-soil system is subdivided into Nth layers along the depth direction in view of the variation of shear modulus or cross-sectional dimension of the pile or differences in soil properties. Then, the vibration displacement solution with undermined constants for any saturated soil layer subjected to the time-harmonic torsional load is obtained by virtue of the separation of variables scheme. To establish the connection of adjacent longitudinal soil layers, the circumferential contact traction at the interface of the adjacent layers is treated as the distributed Winkler subgrade model independent of the radial distance. Then, by utilizing the continuity conditions of the pile-soil system and the method of recursion typically used in the transfer function technique, the torsional impedance of the pile top can be derived in the frequency domain. By virtue of inverse Fourier transform and convolution theorem, the velocity response of an inhomogeneous pile subjected to a transient half-sine exciting torque and embedded in a layered saturated soil is gained in the time domain. Finally, selected numerical results are gained to investigate the influence of typical defects in pile and soil layering on the velocity response of the pile top in the time domain.
\end{abstract}

\section{Introduction}

Pile foundations of machinery, bridges, offshore structures, and towers are commonly subjected to the dynamic torsional loads induced by the eccentricity in applied lateral loads, such as running machinery, moving traffic, wind, earthquake, and impact. Therefore, the analysis of the dynamic response of piles under torsional loads is essential in understanding the distribution of the displacements and forces in piles and can also provide valuable guidelines for dynamic foundation design and nondestructive detection of piles. In the past, various analytical, semianalytical, and numerical methods were proposed to solve the pile-soil dynamic interaction problem.

In the pile-soil dynamic analysis, the key challenge is the theoretical model of the surrounding soil. Over the past several decades, the simplified continuum model was widely utilized to model the surrounding soil, and the corresponding dynamic response of the pile-soil system can be solved by an analytical or semianalytical method. For instance, Militano and Rajapakse [1] investigated the time history of twist angle and vertical displacement at the top end of a pile embedded in a multilayered soil and subjected to transient torsional and axial loads via a semianalytical method. Zhao and Xiang [2] investigated the torsional vibration of pipe pile in unsaturated soil based on the mixture theory. $\mathrm{Wu}$ et al. [3] proposed an analytical solution to calculate the apparent phase velocity of the pipe pile segment with soil plug filling inside based on the additional mass model. Luan et al. [4] presented a new analytical model for calculating the horizontal dynamic impedance of pile groups with arbitrary members connected with a rigid pile cap. Guan et al. [5] investigated the torsional vibration characteristics of tapered pile considering both the compaction 
effect of the pile surrounding soil and the stress diffusion effect of pile end soil. Moreover, based on this simplified continuum model, the analytical solution for the dynamic torsional and vertical response of a radially inhomogeneous soil layer can also be derived (the work of Veletsos and Dotson [6], Dotson and Veletsos [7], Han and Sabin [8], and Zhang et al. [9]). Compared to the simplified continuum model, the continuum model is rigorous in theory and can simulate correctly the mechanism of the soil-structure dynamic interaction. Hence, this rigorous model has been comprehensively applied into the study of the soil-rigid circular disc/footing interaction $[10,11]$ and pile-soil interaction [12-15]. Furthermore, various numerical methods are also employed to study the pile-soil interaction problem. Liu and Novak [16] investigated the dynamic response of a single pile embedded in transversely isotropic layered media using the finite element method (FEM). Tham et al. [17] studied the torsional vibration of an elastic pile embedded in a layered half-space via a coupled FEM-BEM method. Chen et al. [18] proposed a three-dimensional wave-pile-soil coupling FEM to investigate the deformation mechanism of monopile under current and fifth-order Stokes wave. Xu et al. [19] developed a fully coupled dynamic effective-stress FEM to study the effects of the frequency content of input motion and the amplitude of both horizontal and vertical components of input motion on the settlement of the pile group in saturated sand deposits. By virtue of the discrete element method (DEM), Li et al. [20] examined the sand plug behavior inside an open-ended pile considering the pile driving process. Since Biot $[21,22]$ established the general theory of elastic wave propagation in a fluid saturated poroelastic medium, the dynamic response of the buried loads $[23,24]$ and vibration of pile and rigid circular disc in the poroelastic medium were investigated in detail [25-29].

Most of the existing studies are mainly concerned on the vibration characteristics of an intact pile, which is commonly used in the dynamic foundation design. However, in engineering practice, no matter what construction method is employed in pile formation, various defects in pile, such as bulging, necking, and weak concrete, are inevitable, and this kind of defective pile is generally called defective or inhomogeneous pile. It is noted that the defects in a pile could greatly affect the static and dynamic bearing capacities of the pile. Hence, in order to evaluate the potential defects in a pile, the related works were carried out by researchers. For instance, Wu et al. [30] studied the vertical vibration characteristics of a variable cross-section pile and variable modulus pile and gave an important insight into the evaluation of the construction quality of the pile. Liu et al. [31] developed a solution to the torsional response of a multidefective pile in layered soil based on the simplified continuum model and found that shear wave has a special advantage in detecting the shallow defect in a pile.

However, the two abovementioned studies are limited to the purely elastic soil. Therefore, the objective of the present paper is to develop a semianalytical solution to investigate the dynamic response of an inhomogeneous pile embedded in a layered saturated soil. At first, the time-harmonic torsional vibration solution of the pile-soil system is obtained in the frequency domain using the separation of variables scheme and transfer function technique. Then, the velocity response at the pile top is derived by virtue of inverse Fourier transform and convolution theorem. Using the solution developed, the selected numerical results are obtained to analyze the influence of the typical defects in a pile and soil layering on the velocity response of the pile top in the time domain.

\section{Statement of the Boundary-Value Problem}

2.1. Basic Assumptions and Governing Equations. As shown in Figure 1, the problem considered in this paper is the dynamic torsional response of an inhomogeneous elastic pile embedded in a layered saturated soil. Allowing for the variation of shear modulus or cross-sectional dimension of the pile or differences in soil properties of a layered saturated soil, the pile-soil system is subdivided into a total of $N$ segments (layers) numbered by $1,2, \ldots, j, \ldots, N$ from pile tip to pile top. The thickness of the $j$ th $(1 \leq j \leq N)$ soil layer is equal to the length of the $j$ th $(1 \leq j \leq N)$ pile segment and is denoted by $h_{j}$ such that the $j$ th pile segment is completely embedded in the $j$ th soil layer. In view of the symmetry of the torsional vibration problem, the cylindrical coordinate system $(r, \theta, z)$ is adopted in the present study. In order to derive the solution for the present problem, the following assumptions are made during the analysis: (1) The soil is a linear elastic isotropic saturated layer, and the pile is vertical, elastic, end bearing, and circular in cross section. The pile and soil layer properties are assumed to be homogeneous within each segment or layer, respectively, but may change from segment to segment or layer to layer; (2) the pile-soil system is subjected to small deformations and strains during the vibration. The pile has a perfect contact with the surrounding soil during the vibration; (3) the free surface of the soil has no normal and shear stresses, and the soil is infinite in the radial direction; (4) there is no displacement occurring at the bottom of the layer due to the fixed bottom boundary; (5) to establish the connection of adjacent longitudinal soil layers, the circumferential contact traction at the interface of adjacent soil layers is treated as the distributed Winkler subgrade model independent of the radial distance. Moreover, the circumferential contact tractions acting at the $j$ th soil layer due to $(j-1)$ th and $(j+1)$ th soil layers are, respectively, denoted by the distributed reaction coefficients as $k_{\text {st } j}$ and $k_{\mathrm{sbj}}$, which represent the contact stress $\left(\tau_{\theta z}\right)$ per unit of the circumferential displacement along the adjacent soil interface. The reliability of this assumption will be proved in the following section, and the key derivation procedure is illustrated in Figure 2.

The dynamic equilibrium equation of the $j$ th $(1 \leq j \leq N)$ saturated soil subjected to torsional load can be expressed in terms of a cylindrical coordinate system as [26]

$$
\begin{aligned}
G_{s j} \nabla^{2} u_{\theta j}(r, z, t)-\frac{u_{\theta j}(r, z, t)}{r^{2}} G_{s j}= & \rho_{j} \frac{\partial^{2} u_{\theta j}(r, z, t)}{\partial t^{2}} \\
& +\rho_{f j} \frac{\partial^{2} w_{\theta j}(r, z, t)}{\partial t^{2}},
\end{aligned}
$$




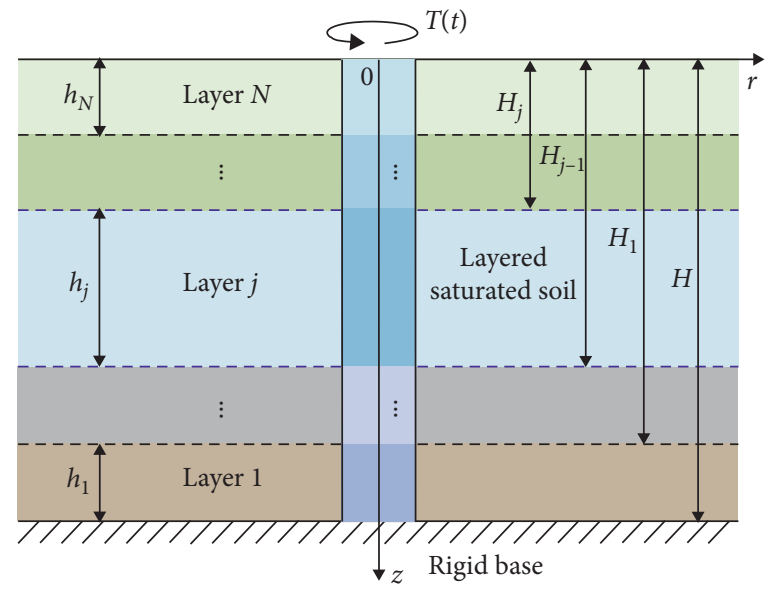

Figure 1: Interaction between an inhomogeneous elastic pile and the layered saturated soil.

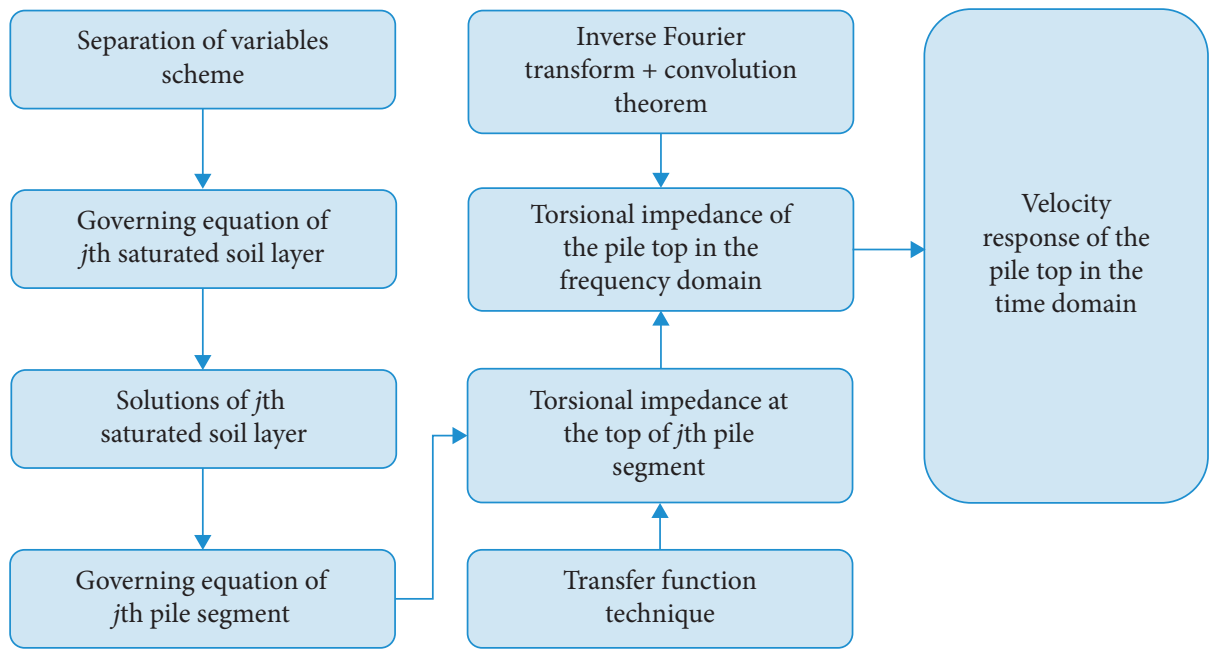

Figure 2: Flow chart of the proposed semianalytical method.

where $u_{\theta j}(r, z, t)$ and $w_{\theta j}(r, z, t)$ are, respectively, the circumferential displacements of the soil skeleton in the $j$ th saturated soil layer and that of the pore fluid relative to the soil skeleton; $\rho_{j}=\left(1-n_{j}\right) \rho_{\mathrm{s} j}+n_{j} \rho_{f j}$ is the bulk density of the $j$ th soil layer; for the $j$ th soil layer, $n_{j}$ is the porosity of the soil; $\rho_{f j}$ is the density of the pore fluid; $G_{\mathrm{s} j}$ and $\rho_{\mathrm{s} j}$ are the shear modulus and density of the soil skeleton, respectively; and $\nabla^{2}=\left(\partial^{2} / \partial r^{2}\right)+(1 / r)(\partial / \partial r)+\left(\partial^{2} / \partial z^{2}\right)$.

According to the study by Zienkiewicz et al. [32], the equation of motion of the pore fluid in a saturated soil can be written as

$$
\frac{\rho_{f j} g}{k_{d j}} \frac{\partial w_{\theta j}(r, z, t)}{\partial t}+\rho_{f j} \frac{\partial^{2} u_{\theta j}(r, z, t)}{\partial t^{2}}+\frac{\rho_{f j}}{n_{j}} \frac{\partial^{2} w_{\theta j}(r, z, t)}{\partial t^{2}}=0,
$$

where $k_{d j}$ denotes the dynamic permeability coefficient containing the viscosity of the liquid; $g$ is the gravitational acceleration.

To gain the solution of the transient response of the pile-soil system, we solve the time-harmonic response first. For the time- harmonic motion, $u_{\theta j}(r, z, t)=u_{\theta j}(r, z) e^{i \omega t}$ and $w_{\theta j}(r, z, t)=w_{\theta j}(r$, $z) e^{i \omega t}, \omega$ is the circular frequency of excitation, and $t$ is the time variable. Equations (1) and (2) can, therefore, be expressed as

$$
\begin{aligned}
& G_{s j} \nabla^{2} u_{\theta j}(r, z)-\frac{u_{\theta j}(r, z)}{r^{2}} G_{s j}=-\rho_{j} \omega^{2} u_{\theta j}(r, z) \\
&-\rho_{f j} \omega^{2} w_{\theta j}(r, z), \\
& \frac{i \rho_{f j} g}{k_{d j}} w_{\theta j}(r, z)-\rho_{f j} \omega u_{\theta j}(r, z)-\frac{\rho_{f j} \omega w_{\theta j}(r, z)}{n_{j}}=0 .
\end{aligned}
$$

Substituting equation (4) into equation (3), the governing equation of the $j$ th saturated soil layer can be written as

$$
\begin{gathered}
\frac{\partial^{2} u_{\theta j}(r, z)}{\partial r^{2}}+\frac{1}{r} \frac{\partial u_{\theta j}(r, z)}{\partial r}+\frac{\partial^{2} u_{\theta j}(r, z)}{\partial z^{2}}-\frac{u_{\theta j}(r, z)}{r^{2}} \\
=-\frac{\omega^{2}}{G_{s j}}\left(\rho_{j}+\frac{n_{j} \omega \rho_{f j}}{i b_{j} / \rho_{f j}-\omega}\right) u_{\theta j}(r, z) .
\end{gathered}
$$


The one-dimensional equation of motion of the $j$ th pile segment subjected to a time-harmonic torsional load can be expressed as

$$
G_{p j} \frac{\partial^{2}\left[\phi_{j}(z) e^{i \omega t}\right]}{\partial z^{2}}+4 \frac{f_{j}(z) e^{i \omega t}}{r_{j}^{2}}=\rho_{p j} \frac{\partial^{2}\left[\phi_{j}(z) e^{i \omega t}\right]}{\partial t^{2}}
$$

where $G_{p j}, \rho_{p j}, r_{j}$, and $\phi_{j}(z)$ are the shear modulus, density, radius, and the twist angle amplitude of the $j$ th pile segment, respectively; $f_{j}(z)$ denotes the amplitude of the contact traction along the $j$ th pile-soil interface.

2.2. The Boundary and Continuity Conditions of the Pile-Soil System. The boundary conditions of the $j$ th soil layer can be written in terms of the local coordinate system as

$$
\begin{aligned}
& u_{\theta j}(r \longrightarrow \infty, z)=0,(1 \leq j \leq N), \\
& \frac{\partial u_{\theta j}(r, z)}{\partial z}-\left.\frac{k_{s t j}}{G_{s j}} u_{\theta j}(r, z)\right|_{z=0}=0, \quad(1 \leq j \leq N), \\
&\left.u_{\theta j}(r, z)\right|_{z=h_{j}}=0, \quad(j=1), \\
& \frac{\partial u_{\theta j}(r, z)}{\partial z}+\left.\frac{k_{s b j}}{G_{s j}} u_{\theta j}(r, z)\right|_{z=h_{j}}=0, \quad(2 \leq j \leq N),
\end{aligned}
$$

where $k_{s t j}$ and $k_{s b j}$ are the distributed reaction coefficients at the top and bottom of the $j$ th soil layer, respectively. It is noted that $k_{s t N}$ is equal to zero due to the free surface of the soil.

The continuity condition of the interface between the $j$ th and $(j+1)$ th $(1 \leq j \leq N-1)$ soil layers can be written as

$$
k_{s t j}=k_{s b(j+1)} \text {. }
$$

The boundary conditions at the base and top of the $j$ th pile segment can be expressed in terms of the local coordinate system as

$$
\begin{aligned}
\left.\frac{\mathrm{d} \phi_{j}(z)}{\mathrm{d} z}\right|_{z=0} & =-\frac{T_{j}}{G_{p j} I_{p j}}, \quad(1 \leq j \leq N), \\
\left.\phi_{j}(z)\right|_{z=h_{j}} & =0, \quad(j=1), \\
\frac{\mathrm{d} \phi_{j}(z)}{\mathrm{d} z}+\left.\frac{\phi_{j}(z) k_{p b j}}{G_{p j} I_{p j}}\right|_{z=h_{j}} & =0, \quad(2 \leq j \leq N),
\end{aligned}
$$

where $T_{j}$ denotes the torque amplitude of the $(j+1)$ th pile segment acting on the top end of the $j$ th pile segment when $1 \leq j \leq N-1$; for the $N$ th pile segment, $T_{N}$ denotes the torque amplitude applied at the pile top; $I_{p j}$ is the polar moment of inertia of the $j$ th pile segment; and $k_{\mathrm{pb} j}$ is the reaction coefficient at the bottom of the $j$ th pile segment.

At the interface between the $j$ th and $(j+1)$ th pile segments $\left(z=H_{j}\right)$, the circumferential displacement and torque of the pile satisfy the following continuity conditions (see Figure 3):

$$
\left\{\begin{array}{l}
\phi_{j}(z)=\phi_{j+1}(z), \quad j=1,2, \ldots N, \\
G_{p j} I_{p j} \frac{d \phi_{j}(z)}{d z}=G_{p(j+1)} I_{p(j+1)} \frac{d \phi_{j+1}(z)}{d z} .
\end{array}\right.
$$

Due to the continuity conditions of the twist angle and torque at the interface of the adjacent pile segment, the torsional impedance is continuous at the corresponding interface. Accordingly, the torsional impedance of the $j$ th pile segment is equal to the reaction coefficient at the base of the $(j+1)$ th pile segment, and the continuity conditions of the $j$ th pile segment can then be written as

$$
k_{T j}=\left.\frac{T_{j}(z)}{\phi_{j}(z)}\right|_{z=H_{j}}=k_{p b(j+1)} .
$$

The continuity conditions of the displacement and stress of the pile-soil interface can expressed as

$$
\begin{gathered}
u_{\theta j}\left(r_{j}, z\right)=\phi_{j}(z) r_{j} \\
f_{j}(z)=\left.\tau_{r \theta j}(r, z)\right|_{r=r_{j}}=\left.G_{s j}\left[\frac{\partial u_{\theta j}(r, z)}{\partial r}-\frac{u_{\theta j}(r, z)}{r}\right]\right|_{r=r_{j}} .
\end{gathered}
$$

\section{Solution of the Pile-Soil System}

3.1. Solution of the Soil Layer. Substituting a single-variable function $u_{\theta j}(r, z)=R_{j}(r) Z_{j}(z)$ into equation (5), we have

$$
\begin{array}{r}
\frac{\mathrm{d}^{2} Z_{j}(z)}{\mathrm{d} z^{2}}+J_{j}^{2} Z_{j}(z)=0, \\
r^{2} \frac{\mathrm{d}^{2} R_{j}(r)}{\mathrm{d} r^{2}}+r \frac{\mathrm{d} R_{j}(r)}{\mathrm{d} r}-\left(1+q_{j}^{2} r^{2}\right) R_{j}(r)=0,
\end{array}
$$

where

$$
q_{j}^{2}=J_{j}^{2}-\frac{\omega^{2}}{G_{s j}}\left(\rho_{j}+\frac{n_{j} \rho_{f j} \omega}{i b_{j} / \rho_{f j}-\omega}\right) .
$$

The general solutions of equation (20) can be expressed as

$$
\begin{aligned}
& Z_{j}(z)=C_{j} \sin \left(J_{j} z\right)+D_{j} \cos \left(J_{j} z\right), \\
& R_{j}(r)=A_{j} K_{1}\left(q_{j} r\right)+B_{j} I_{1}\left(q_{j} r\right),
\end{aligned}
$$

where $I_{1}\left(q_{j} r\right)$ and $K_{1}\left(q_{j} r\right)$ are the modified Bessel functions of the first and second kind of first order, respectively; $A_{j}, B_{j}, C_{j}$, and $D_{j}$ are the constants to be determined by the boundary conditions.

To satisfy the boundary condition given in equation (7), the constant $B_{j}=0$. For the 1 st soil layer (i.e., $j=1$ ), substituting the boundary conditions given in equations (8) and (9) into equation (21) results in

$$
\tan \left(h_{j} J_{j}\right)=\frac{-h_{j} J_{j}}{\bar{k}_{s t j}}, \quad(j=1),
$$

where $\bar{k}_{s t j}=k_{s t j} h_{j} / G_{s j}$ denotes the dimensionless reaction coefficient at the top of the $j$ th soil layer. 


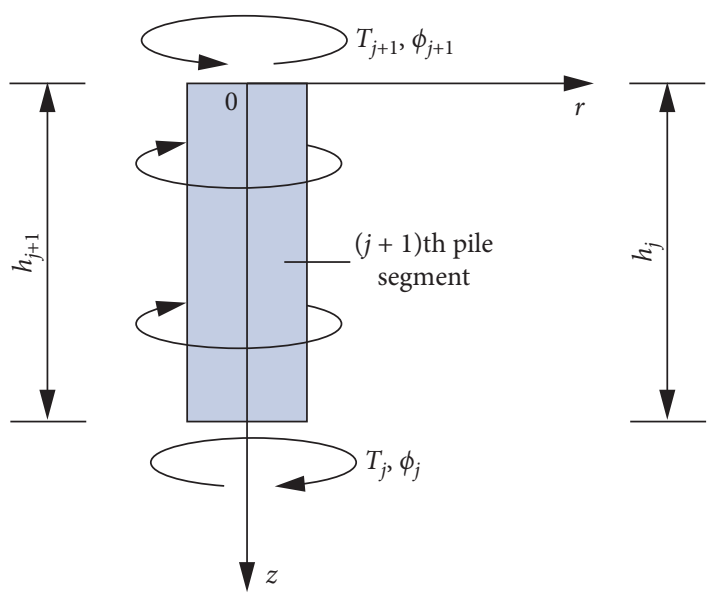

(a)

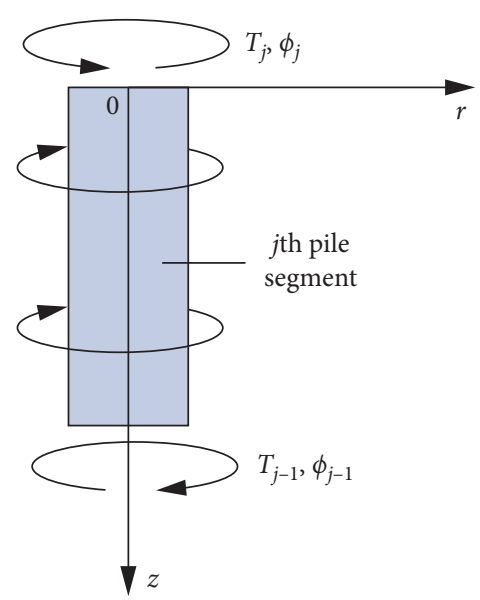

(b)

Figure 3: Pile segment used in derivation of impedance (in the local coordinate system): (a) $(j+1)$ th pile segment and (b) jth pile segment.

For the $j$ th soil layer $(2 \leq j \leq N)$, substituting the boundary conditions given in equations (8) and (10) into equation (21) yields

$$
\tan \left(h_{j} J_{j}\right)=\frac{\left(\bar{k}_{s b j}+\bar{k}_{s t j}\right) h_{j} J_{j}}{\left(h_{j} J_{j}\right)^{2}-\bar{k}_{s b j} \bar{k}_{s t j}}, \quad(2 \leq j \leq N),
$$

where $\bar{k}_{s b j}=k_{s b j} h_{j} / G_{s j}$ denotes the dimensionless reaction coefficients at the base of the $j$ th soil layer. It is noted that $J_{j}$ in equations (22) and (23) can be solved by the numerical method (e.g., bisection method).

Then, the solution of equation (5) can be written as

$$
u_{\theta j}(r, z)=\sum_{m=1}^{\infty} A_{m j} K_{1}\left(q_{m j} r\right) \sin \left(J_{m j} z+\varphi_{m j}\right)
$$

where

$$
\varphi_{m j}=\arctan \left(\frac{J_{m j} h_{j}}{\bar{k}_{s t j}}\right) ; \quad q_{m j}^{2}=J_{m j}^{2}-\frac{\rho_{s j} \omega^{2}}{G_{s j}} .
$$

The shear stress amplitude $\tau_{r \theta j}$ corresponding to equation (24) can be expressed as

$$
\begin{aligned}
\tau_{r \theta j} & =G_{s j}\left[\frac{\partial u_{\theta j}(r, z)}{\partial r}-\frac{u_{\theta j}(r, z)}{r}\right] \\
& =-G_{s j} \sum_{m=1}^{\infty} D_{m j} q_{m j} K_{2}\left(q_{m j} r\right) \sin \left(J_{m j} z+\varphi_{m j}\right),
\end{aligned}
$$

where $K_{2}\left(q_{m j} r\right)$ denotes the modified Bessel functions of the second kind of the second order.

3.2. Solution of the Pile Segment. For the $j$ th $(1 \leq j \leq N)$ pile segment, by utilizing the stress continuity condition that is given in equation (18), substituting equation (26) into equation (6) yields

$$
\begin{aligned}
\phi_{j}(z)= & \alpha_{1 j} \cos \left(\lambda_{j} z\right)+\alpha_{2 j} \sin \left(\lambda_{j} z\right) \\
& +\sum_{m=1}^{\infty} \psi_{m j} \sin \left(J_{m j} z+\varphi_{m j}\right),
\end{aligned}
$$

in which

$$
\psi_{m j}=-\frac{4 G_{s j} q_{m j} K_{2}\left(q_{m j} r_{j}\right) A_{m j}}{G_{p j} r_{j}^{2}\left(J_{m j}^{2}-\lambda_{j}^{2}\right)} ; \quad \lambda_{j}=\sqrt{\frac{\rho_{p j}}{G_{p j}}} \omega,
$$

where $\alpha_{1 j}$ and $\alpha_{2 j}$ are the undetermined coefficients.

By using the displacement continuity condition given in equation (17), substituting equations (24) and (27) into equation (17) results in

$$
\begin{gathered}
r_{j}\left[\alpha_{1 j} \cos \left(\lambda_{j} z\right)+\alpha_{2 j} \sin \left(\lambda_{j} z\right)+\sum_{m=1}^{\infty} \psi_{m j} \sin \left(J_{m j} z+\varphi_{m j}\right)\right] \\
=\sum_{m=1}^{\infty} A_{m j} K_{1}\left(q_{m j} r\right) \sin \left(J_{m j} z+\varphi_{m j}\right) .
\end{gathered}
$$

By invoking the orthogonality of eigenfunctions $\sin \left(J_{m j} z+\varphi_{m j}\right)(m=1,2,3, \ldots)$, multiplying $\sin \left(J_{m j} z+\varphi_{m j}\right)$ on both sides of equation (29), and then, integrating over the interval $z=\left[0, h_{j}\right]$, the undetermined coefficient $A_{m j}$ is found to be

$$
A_{m j}=\frac{1}{L_{m j} E_{m j}} \int_{0}^{h_{j}} P_{j} \sin \left(J_{m j} z+\varphi_{m j}\right) \mathrm{d} z,
$$

where 


$$
\begin{aligned}
& L_{m j}=\int_{0}^{h_{j}} \sin ^{2}\left(J_{m j} z+\varphi_{m j}\right) \mathrm{d} z ; \quad P_{j}=\alpha_{1 j} \cos \left(\lambda_{j} z\right)+\alpha_{2 j} \sin \left(\lambda_{j} z\right) ; \\
& E_{m j}=\frac{1}{r_{j}}\left[K_{1}\left(q_{m j} r_{j}\right)+\frac{4 G_{s j} q_{m j} K_{2}\left(q_{m j} r_{j}\right)}{G_{p j} r_{j}\left(J_{m j}^{2}-\lambda_{j}^{2}\right)}\right] .
\end{aligned}
$$

The amplitude of the twist angle of the $j$ th pile segment is where then given by

$$
\begin{aligned}
\phi_{j}(z)= & \alpha_{1 j}\left[\cos \left(\lambda_{j} z\right)+\sum_{m=1}^{\infty} \xi_{m j} \sin \left(J_{m j} z+\varphi_{m j}\right)\right] \\
& +\alpha_{2 j}\left[\sin \left(\lambda_{j} z\right)+\sum_{m=1}^{\infty} \zeta_{m j} \sin \left(J_{m j} z+\varphi_{m j}\right)\right]
\end{aligned}
$$

$$
\begin{aligned}
\bar{\lambda}_{j} & =\lambda_{j} h_{j} ; \\
\bar{J}_{m j} & =h_{j} J_{m j} ; \\
\bar{q}_{m j} & =h_{j} q_{m j} ; \\
\bar{r}_{j} & =\frac{r_{j}}{h_{j}} ; \\
\bar{\mu}_{j} & =\frac{G_{p j}}{G_{s j}} ; \\
\bar{E}_{m j} & =r_{j} E_{m j} ; \\
\bar{L}_{m j} & =\frac{L_{m j}}{h_{j}} ; \\
v_{m j} & =\frac{-2 \bar{q}_{m j} K_{2}\left(\bar{q}_{m j} \bar{r}_{j}\right)}{\bar{r}_{j} \bar{\mu}_{j}\left(\bar{J}_{m j}^{2}-\bar{\lambda}_{j}^{2}\right) \bar{E}_{m j} \bar{L}_{m j} ;} \\
\xi_{m j} & =v_{m j}\left[\frac{\cos \left(\varphi_{m j}\right)-\cos \left(\bar{J}_{m j}+\bar{\lambda}_{j}+\varphi_{m j}\right)}{\bar{J}_{m j}+\bar{\lambda}_{j}}+\frac{\cos \left(\varphi_{m j}\right)-\cos \left(\bar{J}_{m j}-\bar{\lambda}_{j}+\varphi_{m j}\right)}{\bar{J}_{m j}-\bar{\lambda}_{j}}\right] ; \\
\zeta_{m j} & =v_{m j}\left[\frac{\sin \left(\varphi_{m j}\right)-\sin \left(\bar{J}_{m j}+\bar{\lambda}_{j}+\varphi_{m j}\right)}{\bar{J}_{m j}+\bar{\lambda}_{j}}+\frac{\sin \left(\varphi_{m j}\right)-\sin \left(\bar{J}_{m j}-\bar{\lambda}_{j}+\varphi_{m j}\right)}{\bar{\lambda}_{j}-\bar{J}_{m j}}\right] .
\end{aligned}
$$

Based on the boundary conditions of the $j$ th $(1 \leq j \leq N)$ pile segment, substituting equations (12)-(14) into equation (32), the variables $\alpha_{1 j}$ and $\alpha_{2 j}$ are obtained, and the torsional impedance function at the top end of the $j$ th pile segment can be written in terms of the local coordinate system as

$$
k_{T j}=\frac{T_{j}}{\phi_{j}(z=0)}=\frac{G_{p j} I_{p j}}{r_{j}}\left(-\bar{r}_{j}\right)\left\{\frac{\left(\alpha_{1 j} / \alpha_{2 j}\right) \sum_{m=1}^{\infty} \xi_{m j} \bar{J}_{m j} \cos \left(\varphi_{m j}\right)+\left[\bar{\lambda}_{j}+\sum_{m=1}^{\infty} \zeta_{m j} \bar{J}_{m j} \cos \left(\varphi_{m j}\right)\right]}{\left(\alpha_{1 j} / \alpha_{2 j}\right)\left[1+\sum_{m=1}^{\infty} \xi_{m j} \sin \left(\varphi_{m j}\right)\right]+\sum_{m=1}^{\infty} \zeta_{m j} \sin \left(\varphi_{m j}\right)}\right\},
$$


where

$$
\begin{aligned}
& \frac{\alpha_{1 j}}{\alpha_{2 j}}=-\frac{\sin \left(\bar{\lambda}_{1}\right)+\sum_{m=1}^{\infty} \zeta_{m 1} \sin \left(\bar{J}_{m 1}+\varphi_{m 1}\right)}{\cos \left(\bar{\lambda}_{1}\right)+\sum_{m=1}^{\infty} \xi_{m 1} \sin \left(\bar{J}_{m 1}+\varphi_{m 1}\right)}, \quad(j=1), \\
& \frac{\alpha_{1 j}}{\alpha_{2 j}}=-\frac{\bar{\lambda}_{j} \cos \left(\bar{\lambda}_{j}\right)+\sum_{m=1}^{\infty} \zeta_{m j} \bar{J}_{m j} \cos \left(\bar{J}_{m j}+\varphi_{m j}\right)+\left(h_{j} k_{T(j-1)} / G_{p j} I_{p j}\right)\left[\sin \left(\bar{\lambda}_{j}\right)+\sum_{m=1}^{\infty} \zeta_{m j} \sin \left(\bar{J}_{m j}+\varphi_{m j}\right)\right]}{-\bar{\lambda}_{j} \sin \left(\bar{\lambda}_{j}\right)+\sum_{m=1}^{\infty} \xi_{m j} \bar{J}_{m j} \cos \left(\bar{J}_{m j}+\varphi_{m j}\right)+\left(h_{j} k_{T(j-1)} / G_{p j} I_{p j}\right)\left[\cos \left(\bar{\lambda}_{j}\right)+\sum_{m=1}^{\infty} \xi_{m j} \sin \left(\bar{J}_{m j}+\varphi_{m j}\right)\right]}, \quad(2 \leq j \leq N) .
\end{aligned}
$$

Through further recursion from the 1st pile segment to the $N$ th pile segment, the torsional impedance function at the pile head can be expressed as

$$
k_{T}=k_{T N}=\frac{T_{N}}{\phi_{N}(z=0)}=\frac{G_{p N} I_{p N}}{r_{N}}\left(-\bar{r}_{N}\right)\left\{\frac{\left(\alpha_{1 N} / \alpha_{2 N}\right) \sum_{m=1}^{\infty} \xi_{m N} \bar{J}_{m N} \cos \left(\varphi_{m N}\right)+\left[\bar{\lambda}_{N}+\sum_{m=1}^{\infty} \zeta_{m N} \bar{J}_{m N} \cos \left(\varphi_{m N}\right)\right]}{\left(\alpha_{1 N} / \alpha_{2 N}\right)\left[1+\sum_{m=1}^{\infty} \xi_{m N} \sin \left(\varphi_{m N}\right)\right]+\sum_{m=1}^{\infty} \zeta_{m N} \sin \left(\varphi_{m N}\right)}\right\} .
$$

Based on the definition in [26], the dimensionless tor- where sional impedance at the pile top can be expressed as

$$
k_{T}^{\prime}=\frac{3 T_{N}}{16 G_{s N} r_{N}^{3} \phi(z=0)}=-\frac{3 \pi \bar{\mu}_{N} \bar{r}_{N} R}{32}
$$

$$
R=\frac{\left(\alpha_{1 N} / \alpha_{2 N}\right) \sum_{m=1}^{\infty} \xi_{m N} \bar{J}_{m N} \cos \left(\varphi_{m N}\right)+\left[\bar{\lambda}_{N}+\sum_{m=1}^{\infty} \zeta_{m N} \bar{J}_{m N} \cos \left(\varphi_{m N}\right)\right]}{\left(\alpha_{1 N} / \alpha_{2 N}\right)\left[1+\sum_{m=1}^{\infty} \xi_{m N} \sin \left(\varphi_{m N}\right)\right]+\sum_{m=1}^{\infty} \zeta_{m N} \sin \left(\varphi_{m N}\right)}
$$

The frequency response function of the twist angle of the pile top can be written as

$$
H_{\theta}(\omega)=\frac{1}{k_{T}}=\frac{-r_{N}}{G_{p N} I_{p N}} \frac{1}{\bar{r}_{N}}\left\{\frac{\left(\alpha_{1 N} / \alpha_{2 N}\right)\left[1+\sum_{m=1}^{\infty} \xi_{m N} \sin \left(\varphi_{m N}\right)\right]+\sum_{m=1}^{\infty} \zeta_{m N} \sin \left(\varphi_{m N}\right)}{\left(\alpha_{1 N} / \alpha_{2 N}\right) \sum_{m=1}^{\infty} \xi_{m N} \bar{J}_{m N} \cos \left(\varphi_{m N}\right)+\left[\bar{\lambda}_{N}+\sum_{m=1}^{\infty} \zeta_{m N} \bar{J}_{m N} \cos \left(\varphi_{m N}\right)\right]}\right\} .
$$

Then, the admittance function of angular velocity of the arbitrary point at the pile top can be further expressed as

$$
H_{v}(\omega)=i \omega r_{s} H_{\theta}(\omega)=\frac{r_{s}}{v_{p s N} I_{p N} \rho_{p N}} H_{v}^{\prime}(\omega)
$$

where $r_{s}$ denotes the radial distance to the arbitrary point, and it is commonly selected as $r_{0}$ (i.e., the radius of the homogeneous pile); the dimensionless admittance function of angular velocity of the pile top is

$$
H_{v}^{\prime}(\omega)=-i \bar{\lambda}_{N}\left\{\frac{\left(\alpha_{1 N} / \alpha_{2 N}\right)\left[1+\sum_{m=1}^{\infty} \xi_{m N} \sin \left(\varphi_{m N}\right)\right]+\sum_{m=1}^{\infty} \zeta_{m N} \sin \left(\varphi_{m N}\right)}{\left(\alpha_{1 N} / \alpha_{2 N}\right) \sum_{m=1}^{\infty} \xi_{m N} \bar{J}_{m N} \cos \left(\varphi_{m N}\right)+\left[\bar{\lambda}_{N}+\sum_{m=1}^{\infty} \zeta_{m N} \bar{J}_{m N} \cos \left(\varphi_{m N}\right)\right]}\right\}
$$


By applying the inverse Fourier transform into equation (40), the response function of unit pulse torque in the time domain can be written as

$$
h(t)=\operatorname{IFT}\left[H_{v}(\omega)\right]=\frac{1}{2 \pi} \int_{-\infty}^{+\infty} \frac{r_{s}}{v_{p s N} I_{p N} \rho_{p N}} H_{v}^{\prime}(\omega) e^{i \tilde{\omega} \bar{t}} \mathrm{~d} \widetilde{\omega},
$$

where $\widetilde{\omega}=\omega T_{c}$ and $T_{c}=\sum_{j=1}^{N} h_{j} / v_{p s j}$ denote the nondimensional frequency and propagation time of elastic shear wave propagating from the pile top to pile tip, respectively; $\bar{t}=t / T_{c}$ is the nondimensional time variable.

If the Fourier transform of the arbitrary exciting torque $T(t)$ acting at the pile top is denoted by $T(\omega)$, then the velocity response of the pile top in the time domain is given via the inverse Fourier transform and convolution theorem as $V(t)=T(t) * h(t)=\operatorname{IFT}\left[T(\omega) H_{v}(\omega)\right]$. In particular, when the exciting torque is a half-sine pulse, we have

$$
T(t)= \begin{cases}T_{\max } \sin \frac{\pi t}{t_{0}}, & t<t_{0}, \\ 0, & t \geq t_{0},\end{cases}
$$

where $t_{0}$ and $T_{\max }$ denote the duration of the impulse and the maximum amplitude of the exciting torque, respectively.

Then, the velocity response of the pile top in the time domain can be written as

$$
V(t)=T_{\max } I F T\left[\frac{r_{s}}{v_{p s N} I_{p N} \rho_{p N}} H_{v}^{\prime}(\omega) \frac{\pi t_{0}}{\pi^{2}-t_{0}^{2} \omega^{2}}\left(1+e^{-i \omega t_{0}}\right)\right] .
$$

It is noted that the infinite integral involved in equation (44) can be numerically determined with finite terms (e.g., the lower and upper limits of the integral are from -4000 to 4000).

To facilitate analysis, it is useful to introduce the normalized velocity response

$$
V^{\prime}(t)=\frac{V(t)}{\max [V(t)]},
$$

where $\max [V(t)]$ denotes the maximum value of $V(t)$.

\section{Numerical Results and Discussion}

4.1. Comparison with Other Solutions. It is noted that the existed solutions for an elastic pile subjected to a harmonic torsional loading and embedded in layered soil neglect the gradient of the shear stress $\tau_{\theta z}$ in the depth direction $[1,31]$, thus resulting in inevitable error in the dynamic response. However, when considering the effect of the shear stress $\tau_{\theta z}$, it is difficult to propose a rigorous solution due to the unknown continuity conditions of the adjacent soil layers. Accordingly, the reaction coefficient of adjacent soil layers is proposed in this paper to establish the connection of the adjacent soil layers and to derive the corresponding solution. Apparently, the value of the reaction coefficient has an important influence on the reliability and applicability of the developed solution. In the following section, unless otherwise specified, the pile properties are $\rho_{p j}=2300 \mathrm{~kg} / \mathrm{m}^{3}$, $v_{p s j}=2450 \mathrm{~m} / \mathrm{s}, \quad G_{p j}=1.38 \times 10^{10} \mathrm{~Pa}, \quad E_{p j}=3.31 \times 10^{10} \mathrm{~Pa}$, $r_{j}=r_{0}=0.4 \mathrm{~m}(j=1,2, \ldots, N)$, and the Poisson's ratio is 0.2 ; the soil properties are $\rho_{s j}=2650 \mathrm{~kg} / \mathrm{m}^{3}, \rho_{f j}=1000 \mathrm{~kg} / \mathrm{m}^{3}$, $n_{j}=0.4$, and $G_{s j}=1.38 \times 10^{7} \mathrm{~Pa}(j=1,2, \ldots, N)$.

To verify the solution developed in the present paper, the pile-soil layer is divided into 3 layers $(N=3)$, and the pile and soil are assumed to be homogeneous. Figure 4 shows the comparison of the torsional impedance of the present study with that of Wang et al. [26]. In Figure 4, $\operatorname{Re}($ ) and $\operatorname{Im}($ ) denote the real and imaginary parts of the physical quantity, respectively. It can be seen from Figure 4 that the real and imaginary parts of the torsional impedance of the present solution are close to those of Wang et al. [26] when $\bar{k}_{s t j}$ (the distributed reaction coefficient at the top of the $j$ th soil layer) is within the range from 0.001 to 0.1 . When $\bar{k}_{\text {st } j}=10$, the real part of the torsional impedance of the present solution has an obvious error in the relatively low frequency range when compared to the rigorous solution. However, the error of the torsional impedance of the present solution is slight, as the excitation frequency is in the higher frequency range. This result indicates that $\bar{k}_{s t j}$ has a negligible influence on the torsional impedance in the higher frequency range.

Figure 5 shows the comparison of the normalized velocity response of a homogeneous pile obtained from the present study with that of Wang et al. [26]. It can be observed from Figure 5 that the velocity response curve of the present solution agrees well with that of Wang et al. [26] when $\bar{k}_{s t j}$ changes from 0.001 to 10 . This is due to the face that the majority of data used in the calculation of the velocity response belongs to high frequency data, and $\bar{k}_{s t j}$ has a negligible influence on the dynamic response in the high frequency range. Consequently, the influence of $\bar{k}_{s t j}$ on the velocity response is negligible, and $\bar{k}_{s t j}$ is fixed at 0.01 in the following analysis.

4.2. Influence of the Neck Width and Blub Width. In practical engineering, cast-in-place piles are widely used, and the corresponding defect can generally be modelled as pile with a neck, pile with a bulb, and pile with a weak concrete segment (see Figure 6). As shown in Figure 6, it is the typically inhomogeneous pile with one defect, and the pile can be divided into 3 segments. $W_{\mathrm{RN}}$ (neck width) and $W_{\mathrm{RB}}$ (bulb width) denote the radii of the neck and bulb, respectively. $L_{\mathrm{E}}$ and $L_{\mathrm{L}}$ denote, respectively, the embedment depth and length of the defect. In addition, $r_{0}$ is the radius of a homogeneous pile.

Figure 7 shows influence of the neck width $\left(W_{\mathrm{RN}}\right)$ on the normalized velocity response of the pile top. It can be seen from Figure 7 that compared to the homogeneous pile, the first reflected signal (i.e., RS1) of the neck arrives at the pile top when $t=t_{\mathrm{RS} 1}=0.00284 \mathrm{~s}\left(t_{\mathrm{RS} 1}\right.$ is the time of arrival of the first reflected signal) and, thus, the calculated $L_{\mathrm{E}}=v_{p s} \times t_{\mathrm{RS} 1} /$ $2=3.48 \mathrm{~m}$ with error $=0.57 \%\left(v_{p s}\right.$ is the one-dimensional elastic shear wave velocity in a homogeneous pile and taken as $2450 \mathrm{~m} / \mathrm{s}$ ). The main reason for the error is the fact that the boundary of reflected signal is difficult to accurately determine in theory. When the time is approximately equal 


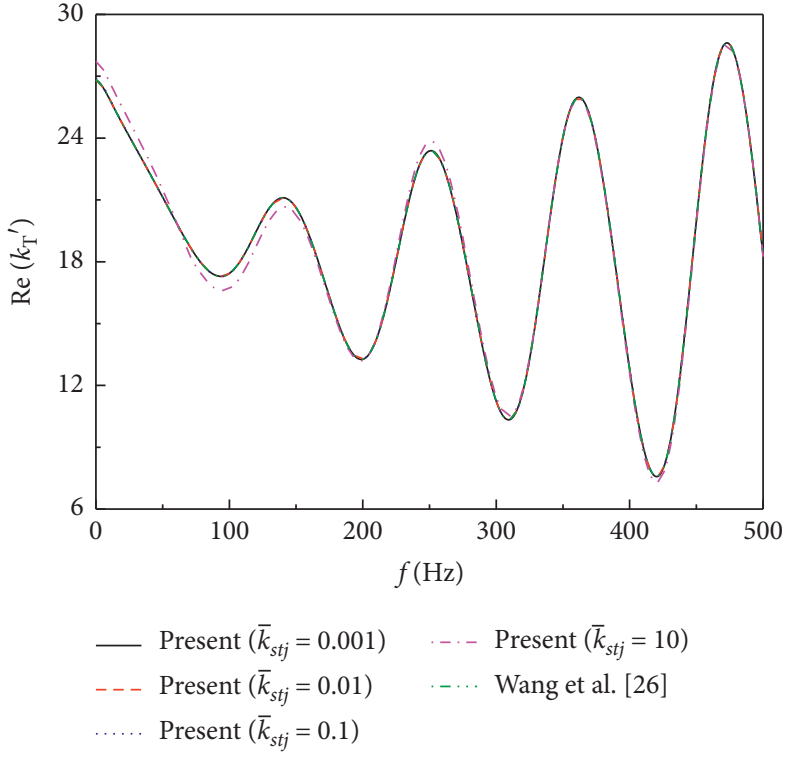

(a)

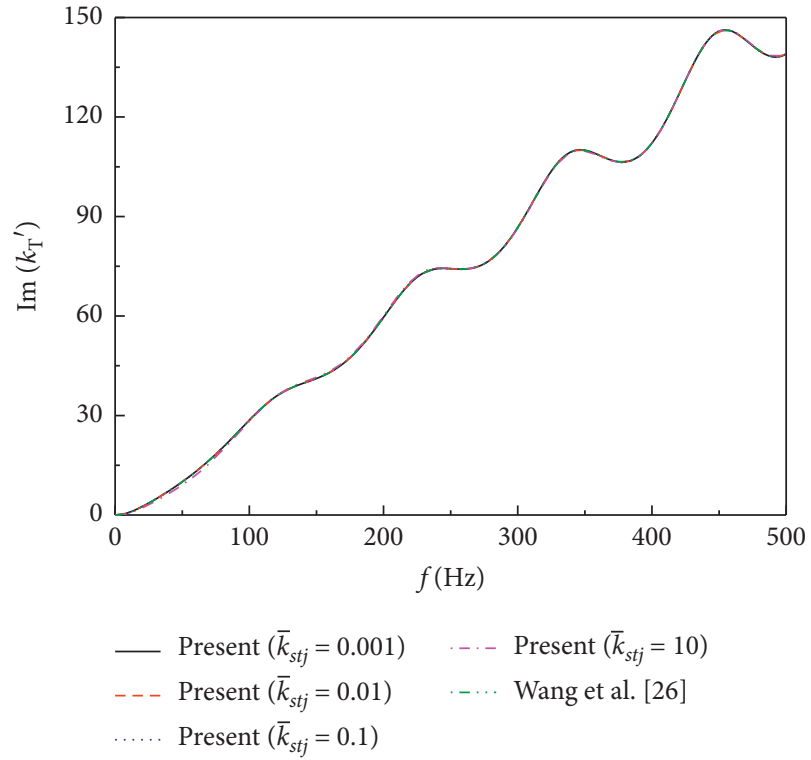

(b)

FIgURE 4: Comparison of the torsional impedance of a homogeneous pile embedded in a homogeneous saturated soil $\left(N=3, h_{1}=7 \mathrm{~m}\right.$, $h_{2}=0.5 \mathrm{~m}$, and $\left.h_{3}=3.5 \mathrm{~m}\right)$.

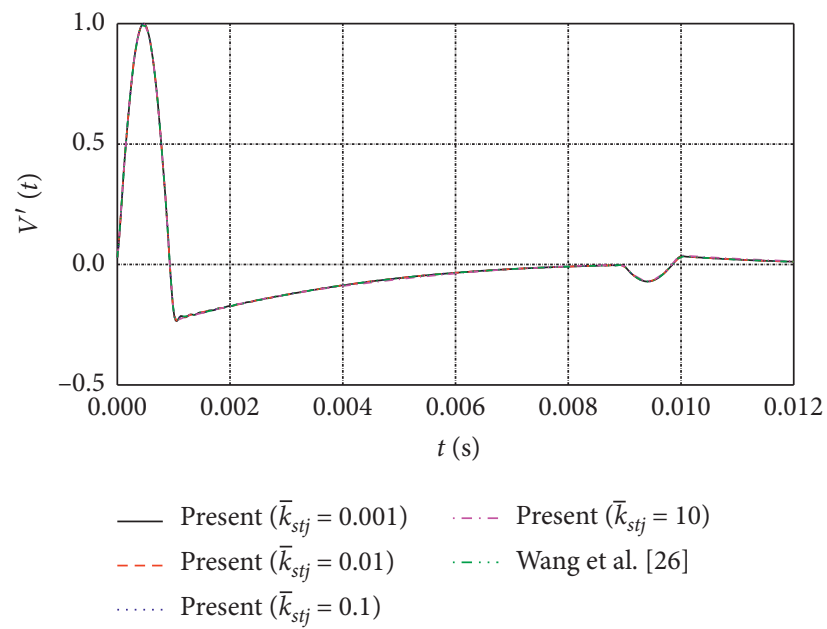

Figure 5: Comparison of the normalized velocity response of for a homogeneous pile embedded in a homogeneous saturated soil $(N=3$, $h_{1}=7 \mathrm{~m}, h_{2}=0.5 \mathrm{~m}$, and $\left.h_{3}=3.5 \mathrm{~m}\right)$.

to two times $t_{\mathrm{RS} 1}$, the second reflected signal (i.e., RS2) of the neck arrives at the pile top with the corresponding amplitude of the reflected signal decreasing markedly due to the energy dissipation in the propagating process. The reflected signal from the pile end (i.e., RSE) arrives at the pile top when $t=t_{\mathrm{RSE}}=0.00899 \mathrm{~s}$ and, thus, $H=v_{p s} \times t_{\mathrm{RSE}} / 2=11.01 \mathrm{~m}$ with error $=0.09 \%$. It is also observed from Figure 7 that the phase of RS1 is the same as that of the incident pulse first and then opposite to that of the incident pulse. The phase of the RSE is opposite to that of the incident pulse for the fixed bottom boundary. Moreover, the amplitude of the RS1 increases markedly with the decrease of $W_{\mathrm{RN}}$. This result indicates that the amplitude of the reflected signal increases with increasing degree of defect.
Figure 8 depicts the influence of the bulb width $\left(W_{\mathrm{RB}}\right)$ on the normalized velocity response of the pile top. It can be seen from Figure 8 that compared to the homogeneous pile, $\mathrm{RS} 1$ arrives at the pile top when $t=t_{\mathrm{RS} 1}=0.00284 \mathrm{~s}$ and, thus, $L_{\mathrm{E}}=v_{p s} \times t_{\mathrm{RS} 1} / 2=3.48 \mathrm{~m}$ with error $=0.57 \%$. When the time is approximately equal to two times $t_{\mathrm{RS} 1}$, RS2 arrives at the pile top and the amplitude of RS2 decreases markedly because of the energy dissipation. RSE arrives at the pile top when $t=t_{\mathrm{RSE}}=0.00899 \mathrm{~s}$ and, thus, $H=v_{p s} \times t_{\mathrm{RSE}} / 2=11.01 \mathrm{~m}$ with error $=0.09 \%$. It is also observed from Figure 8 that the phase of RS1 is the opposite to that of the incident pulse first and then the same with that of the incident pulse. However, the phase of RS2 is the same as that of the incident pulse. Moreover, the amplitude of RS1 increases markedly with the 


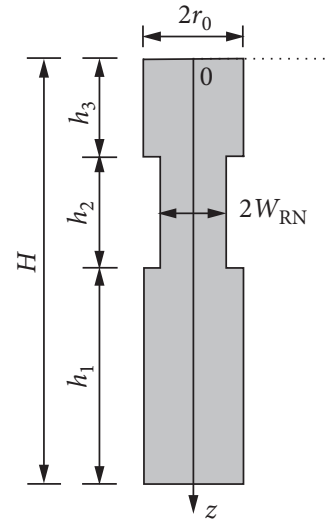

(a)

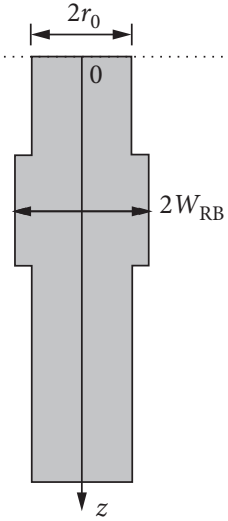

(b)

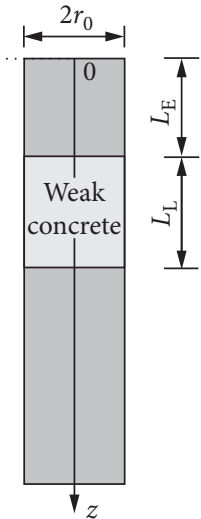

(c)
Figure 6: Geometry of a typically inhomogeneous pile with one defect: (a) pile with a neck; (b) pile with a bulb; and (c) pile with a weak concrete segment.

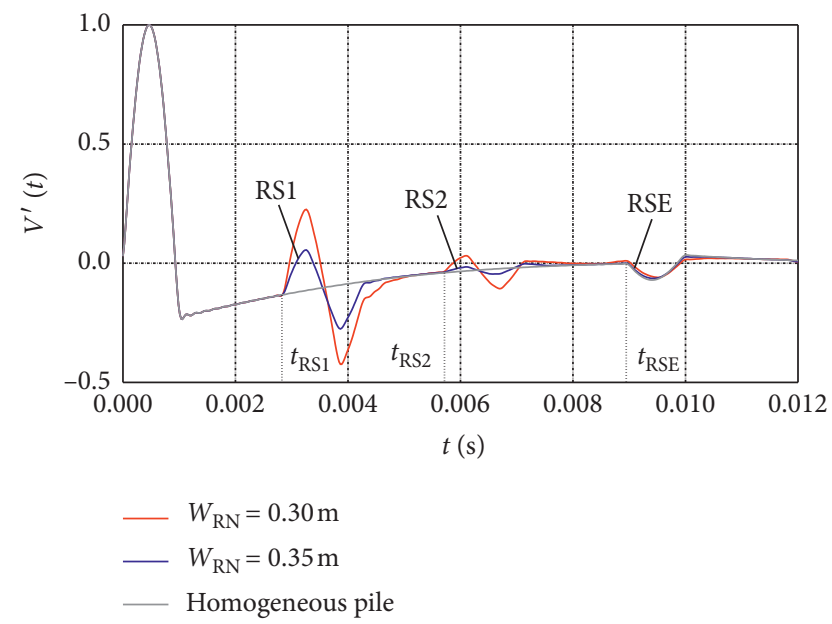

FIGURE 7: Influence of the neck width $\mathrm{W}_{\mathrm{RN}}$ on the normalized velocity response of the pile top $\left(N=3, h_{1}=7=\mathrm{m}, L_{\mathrm{L}}=h_{2}=0.5 \mathrm{~m}\right.$, and $L_{\mathrm{E}}=h_{3}=3.5 \mathrm{~m}$ ).

increase of $W_{\mathrm{RB}}$, which indicates that higher amplitude of the reflected signal is associated with larger value of the bulb width.

4.3. Effect of Weak Concrete. Generally, if the pile has a weak concrete segment, the shear modulus of the pile will decrease greatly. Hence, we use the shear wave velocity to simulate the weak concrete in theory. The influence of the shear wave velocity in the weak concrete segment $\left(v_{p s 2}\right)$ on the normalized velocity response of the pile top is shown in Figure 9. The changing trend of the velocity response at the top end of a pile with a weak concrete segment is similar to that of the pile with a neck. When $t=t_{\mathrm{RS} 1}=0.00284 \mathrm{~s}$, RS1 arrives at the pile top and $L_{\mathrm{E}}=v_{p s} \times t_{\mathrm{RS} 1} / 2=3.48 \mathrm{~m}$ with error $=0.57 \%$. When $t$ is approximately equal to two times $t_{\mathrm{RS}}$, RS2 arrives at the pile top. However, compared to the defect of neck, the amplitude of RS2 is small. The phase of RS1 is opposite to

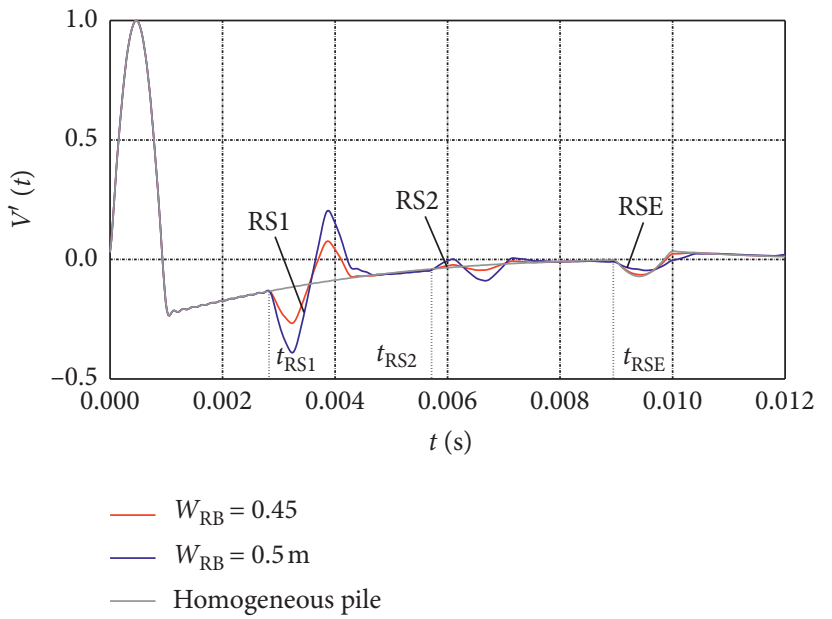

Figure 8: Influence of the bulb width $W_{\mathrm{RB}}$ on the normalized velocity response of the pile top $\left(N=3, h_{1}=7 \mathrm{~m}, L_{\mathrm{L}}=h_{2}=0.5 \mathrm{~m}\right.$, and $L_{\mathrm{E}}=h_{3}=3.5 \mathrm{~m}$ ).

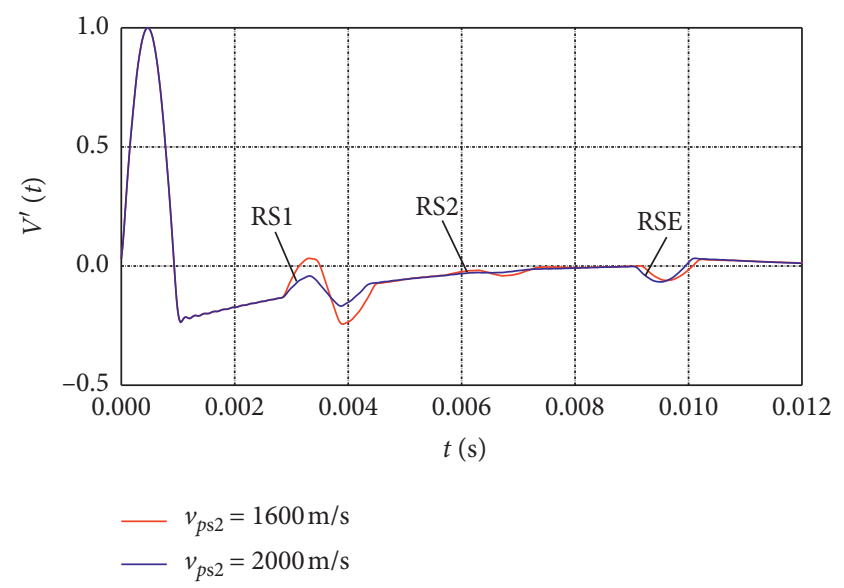

FIGURE 9: Influence of shear wave velocity in weak concrete on the normalized velocity response of the pile top $\left(N=3, h_{1}=7 \mathrm{~m}\right.$, $L_{\mathrm{L}}=h_{2}=0.5 \mathrm{~m}$, and $\left.L_{\mathrm{E}}=h_{3}=3.5 \mathrm{~m}\right)$.

that of the incident pulse first and then the same as that of incident pulse. The amplitude of RS1 increases markedly with the decrease of $v_{p s 2}$. Furthermore, the time of arrival of RSE increases obviously with the decrease of $v_{p s 2}$. This result indicates that RSE will need much more time to arrive at the pile top as $v_{p s 2}$ decreases. As a result, the estimated pile length will be longer than the actual length. For instance, when $t=t_{\mathrm{RSE}}=0.00918 \mathrm{~s}$, RSE of the pile with $v_{p s 2}=1600 \mathrm{~m} / \mathrm{s}$ arrives at the pile top and the pile length can be estimated as $H=v_{p s} \times t_{\mathrm{RSE}} / 2=11.25 \mathrm{~m}$ with error $=2.27 \%$.

4.4. Effect of Length of the Defect. Figure 10 portrays the influence of the neck length on the normalized velocity response of the pile top. It can be seen from Figure 10 that the neck length has marked influence on the velocity response of the pile top. As the neck length increases from $0.5 \mathrm{~m}$ to $2.0 \mathrm{~m}$, the width of RS1 and RS2 shows an obvious 


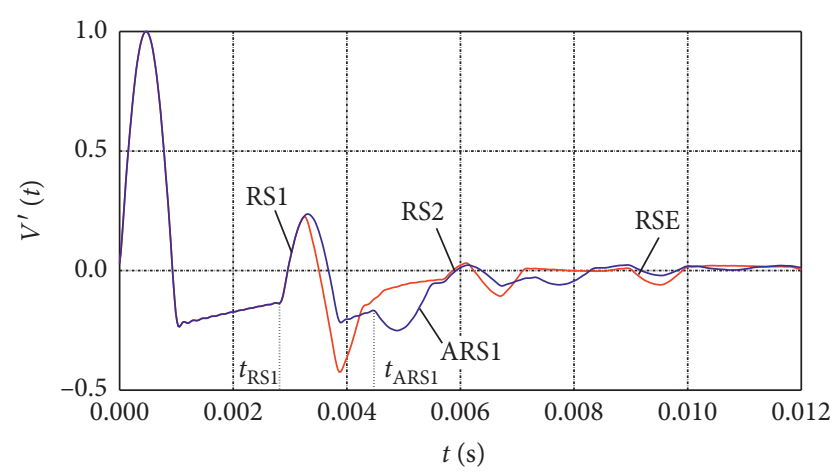

$-L_{\mathrm{L}}=0.5 \mathrm{~m}$

$-L_{\mathrm{L}}=2.0 \mathrm{~m}$

FIGURE 10: Influence of neck length on the normalized velocity response of the pile top $\left(N=3, W_{\mathrm{RN}}=0.3 \mathrm{~m}\right.$, and $\left.L_{\mathrm{E}}=h_{3}=3.5 \mathrm{~m}\right)$.

increase, and another reflected signal (ARS1) arrives at the pile top when $t=0.00448 \mathrm{~s}$. It should be mentioned again that there exist two abrupt changes in the cross-sectional dimension for a pile with a neck, one is at the top of the neck, and the other is at the bottom of the neck. When the neck length is long enough and the shear wave velocity is relatively slow, the reflected signal of these two abrupt changes does not overlap in the time domain. Furthermore, the embedded depth corresponding to the bottom of the neck is $5.5 \mathrm{~m}$, and the corresponding calculated depth is $0.00448 \times 2450 / 2=5.49 \mathrm{~m}$ with error $=0.18 \%$.

Figure 11 shows the influence of the blub length on the normalized velocity response of the pile top. It can be seen from Figure 11 that the blub length has marked influence on the velocity response of the pile top. The width of RS1 and RS2 also increases with the increase of blub length. For the pile with $L_{\mathrm{L}}=2.0 \mathrm{~m}$, another obvious reflected signal (ARS1) arrives when $t=t_{\mathrm{ARS} 1}=0.00448 \mathrm{~s}$, and the phase of ARS1 is the same as that of the incident impulse. It is worth noting that this signal is easy to be identified as a defect by mistake due to the similarity with the reflected signal of the defect.

Figure 12 shows the influence of the weak concrete length on the normalized velocity response of the pile top. It can be seen from Figure 12 that the influence of the weak concrete length on the velocity response is similar to that of the neck length. The width of RS1 and RS2 also increases with the increase of the weak concrete length, and there exists an individual ARS1 when $L_{\mathrm{L}}=2.0 \mathrm{~m}$. Moreover, the time of arrival of RSE shows a marked increase with the increase of $L_{\mathrm{L}}$.

4.5. Influence of Soil Layering. In some cases, the soil properties may change greatly in certain embedment depth. In order to explicitly identify the influence of the variation of the surrounding soil properties, the pile is assumed to be homogeneous, and three cases of soil properties are investigated. Case 1: the soil has a stiff interlayer with $G_{s 2}=5.52 \times 10^{7} \mathrm{~Pa}$ (i.e., $G_{s 1} / G_{s 2}=0.25$ ); Case 2: the soil is homogeneous with $G_{s 2}=1.38 \times 10^{7} \mathrm{~Pa}$ (i.e., $G_{s 1} / G_{s 2}=1.0$ ); and Case 3: the soil has a soft interlayer with

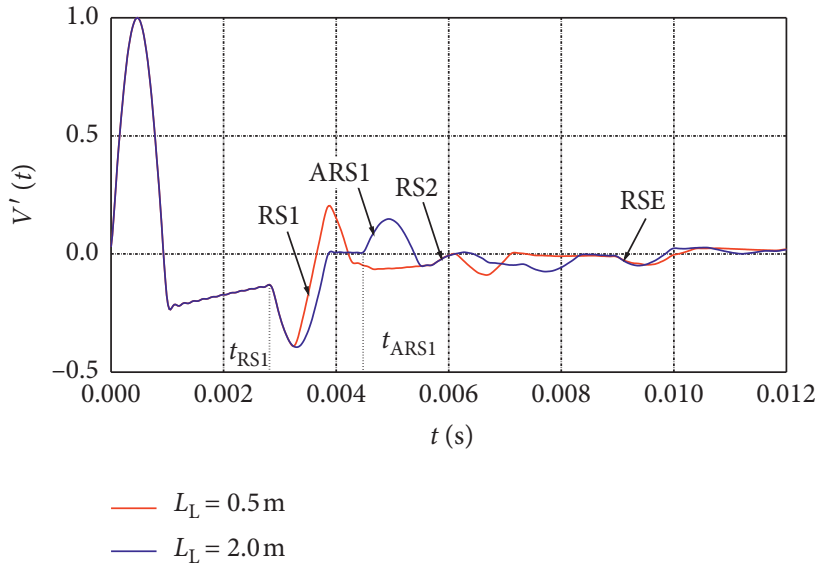

FIgURE 11: Influence of bulb length on the normalized velocity response of the pile top $\left(N=3, W_{\mathrm{RB}}=0.45 \mathrm{~m}\right.$, and $\left.L_{\mathrm{E}}=h_{3}=3.5 \mathrm{~m}\right)$.

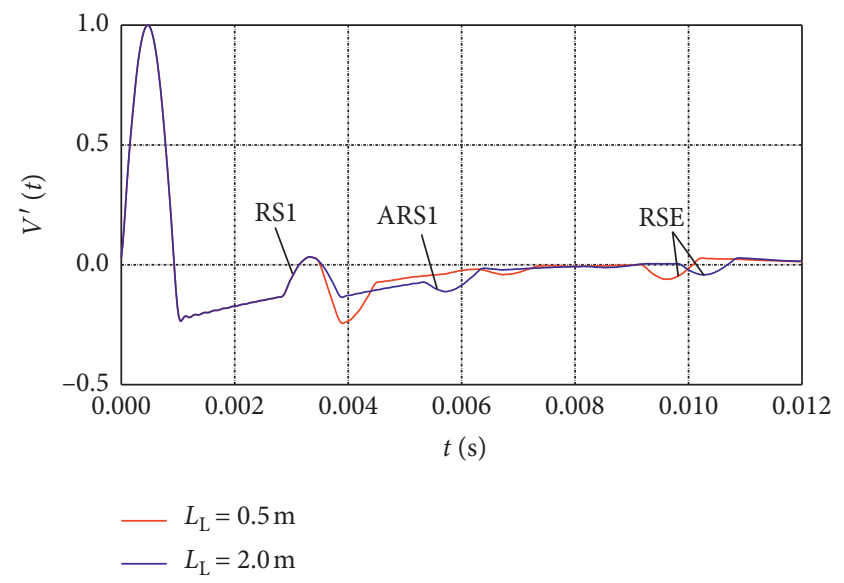

FIGURE 12: Influence of weak concrete length on the normalized velocity response of the pile top $\left(N=3, v_{p s 2}=1600 \mathrm{~m} / \mathrm{s}\right.$, and $\left.L_{\mathrm{E}}=h_{3}=3.5 \mathrm{~m}\right)$.

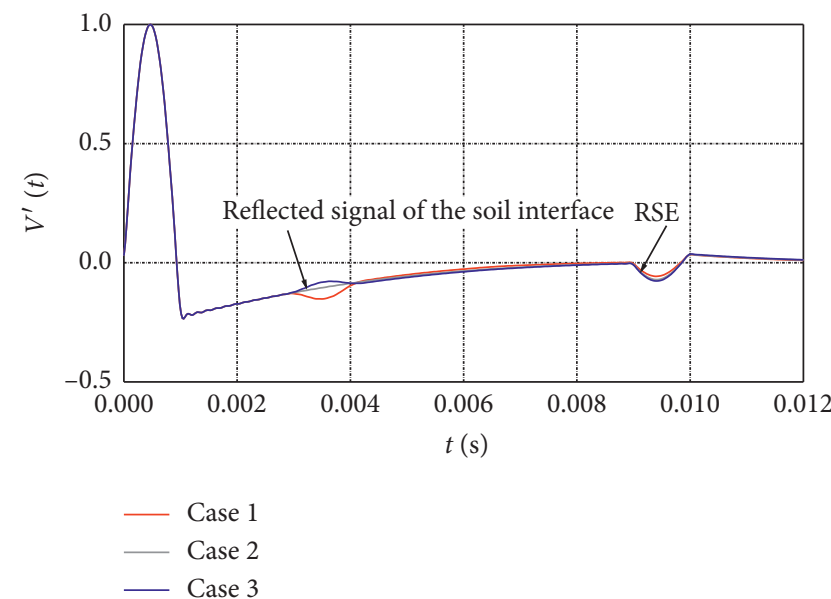

FIGURE 13: Influence of soil layering on the normalized velocity response of the pile $\left(N=3, h_{1}=7 \mathrm{~m}, h_{2}=0.5 \mathrm{~m}\right.$, and $\left.h_{3}=3.5 \mathrm{~m}\right)$. 
$G_{s 2}=3.45 \times 10^{6} \mathrm{~Pa}$ (i.e., $G_{s 1} / G_{s 2}=4$ ). It can be seen from Figure 13 that the phase of the reflected signal of the interface of adjacent soil layers is, respectively, opposite to and same as that of the incident pulse for Case 1 and Case 3 . However, when the soil is homogeneous, the velocity curve is smooth and the reflected signal of the soil interface does not emerge. It is also observed from Figure 13 that the shape of the reflected signal induced by the soil with a soft or stiff interlayer is in a half-sine form, which is obviously different from that of the defect. Moreover, the amplitude of the reflected signal induced by the soil interface is much lower than that of the defect.

\section{Conclusions}

Based on Biot's poroelastodynamic theory, we derive a semianalytical solution for the dynamic response of an inhomogeneous elastic pile embedded in a multilayered saturated soil and subjected to a transient torsional load. The time-harmonic torsional vibration solution of the pile-soil system is derived first in the frequency domain. Then, the time domain solution corresponding to the pile under transient torsional load is gained by virtue of inverse Fourier transform and convolution theorem. After validating the accuracy and reliability of the proposed reaction coefficient, selected numerical results are gained to investigate the influence of the typical defects in pile and soil layering on the velocity response in the time domain. The main conclusions are as follows:

(1) For the end-bearing pile, no matter it is homogeneous or inhomogeneous, the phase of the reflected signal from the pile end is opposite to that of the incident pulse.

(2) For a pile with a neck, the phase of the first and second reflected signals of the defect is the same as that of the incident pulse. The amplitude of reflected signal increases greatly with the decrease of the neck width. The width of reflected signal increases with the increase of the neck length.

(3) For a pile with a bulb, the phase of the first reflected signal is opposite to that of the incident pulse. However, the phase of the second reflected signal is the same as that of the incident pulse. The amplitude of the reflected signal increases greatly with the increase of bulb width.

(4) The characteristic of the reflected signal of the pile with a weak concrete segment is similar to that of the pile with a neck. Furthermore, as the length of the defect (neck, bulb, or weak concrete segment) is long enough, the reflected signal of the upper and lower interfaces of the defect will not overlap in the time domain.

(5) The abrupt change in adjacent soil layer properties has certain influence on the velocity response of the pile top. The shape of the reflected signal of the soil with a soft or stiff interlayer is in a half-sine form, and the amplitude of the reflected signal of soil interface is much lower than that of the defect.

\section{Data Availability}

The data in Figures 4-5 and 7-13 used to support the findings of this study are available from the corresponding author upon request.

\section{Conflicts of Interest}

The authors declare that they have no conflicts of interest.

\section{Acknowledgments}

This research was supported by the National Natural Science Foundation of China (Grant nos. 51878619 and 52078465).

\section{References}

[1] G. Militano and R. K. N. D. Rajapakse, "Dynamic response of a pile in a multi-layered soil to transient torsional and axial loading," Géotechnique, vol. 49, no. 1, pp. 91-109, 1999.

[2] F. Y. Zhao and P. Xiang, "Analysis of Rotary vibration of rigid friction pipe pile in unsaturated soil," Mathematical Problems in Engineering, vol. 2020, Article ID 280757, 12 pages, 2020.

[3] W. Wu, H. Liu, X. Yang et al., "New method to calculate apparent phase velocity of open-ended pipe pile," Canadian Geotechnical Journal, vol. 57, no. 1, pp. 127-138, 2020.

[4] L. Luan, C. Zheng, G. P. Kouretzis, and X. Ding, "Dynamic analysis of pile groups subjected to horizontal loads considering coupled pile-to-pile interaction," Computers and Geotechnics, vol. 117, Article ID 103276, 2020.

[5] W.-j. Guan, W.-b. Wu, G.-s. Jiang, J. L. Chin, and G.-d. Deng, "Torsional dynamic response of tapered pile considering compaction effect and stress diffusion effect," Journal of Central South University, vol. 27, no. 12, pp. 3839-3851, 2020.

[6] A. S. Veletsos and K. W. Dotson, "Vertical and torsional vibration of foundations in inhomogeneous media," Journal of Geotechnical Engineering, vol. 114, no. 9, pp. 1002-1021, 1988.

[7] K. W. Dotson and A. S. Veletsos, "Vertical and torsional impedances for radially inhomogeneous viscoelastic soil layers," Soil Dynamics and Earthquake Engineering, vol. 9, no. 3, pp. 110-119, 1990.

[8] Y. C. Han and G. C. W. Sabin, "Impedances for radially inhomogeneous viscoelastic soil media," Journal of Engineering Mechanics, vol. 121, no. 9, pp. 939-947, 1995.

[9] Y. P. Zhang, X. Y. Yang, W. B. Wu, M. H. El Naggar, G. S. Jiang, and R. Z. Liang, "Torsional complex impedance of pipe pile considering pile installation and soil plug effect," Soil Dynamics and Earthquake Engineering, vol. 131, Article ID 106010, 2020.

[10] E. Pan, H. Liu, and Z. Zhang, "Vertical and torsional vibrations of a rigid circular disc on a transversely isotropic and layered half-space with imperfect interfaces," Soil Dynamics and Earthquake Engineering, vol. 113, pp. 442-453, 2018.

[11] Z. Zhang and E. Pan, "Coupled horizontal and rocking vibrations of a rigid circular disc on a transversely isotropic and layered half-space with imperfect interfaces," International Journal of Solids and Structures, vol. 165, pp. 176-191, 2019.

[12] R. K. N. D. Rajapakse, A. H. Shah, and S. K. Datta, “Torsional vibrations of elastic foundations embedded in an elastic halfspace," Earthquake Engineering \& Structural Dynamics, vol. 15, no. 3, pp. 279-297, 1987. 
[13] R. K. N. D. Rajapakse and A. H. Shah, "Impedance curves for an elastic pile," Soil Dynamics and Earthquake Engineering, vol. 8, no. 3, pp. 145-152, 1989.

[14] Z. Li and Y. F. Gao, "Torsional vibration of a large-diameter pipe pile embedded in inhomogeneous soil," Ocean Engineering, vol. 172, pp. 737-758, 2019.

[15] L. Luan, X. Ding, C. Zheng, G. Kouretzis, and Q. Wu, "Dynamic response of pile groups subjected to horizontal loads," Canadian Geotechnical Journal, vol. 57, no. 4, pp. 469-481, 2020.

[16] W. M. Liu and M. Novak, "Dynamic response of single piles embedded in transversely isotropic layered media," Earthquake Engineering and Structural Dynamics, vol. 23, no. 11, pp. 1239-1257, 1994.

[17] L. G. Tham, Y. K. Cheung, and Z. X. Lei, "Torsional dynamic analysis of single piles by time-domain BEM," Journal of Sound and Vibration, vol. 174, no. 4, pp. 505-519, 1994.

[18] L. B. Chen, X. Y. Yang, L. C. Li et al., "Numerical analysis of the deformation performance of monopile under wave and current load," Energies, vol. 13, no. 23, p. 6431, 2020.

[19] L. Y. Xu, C. X. Song, W. Y. Chen, F. Cai, Y. Y. Li, and G. X. Chen, "Liquefaction-induced settlement of the pile group under vertical and horizontal ground motions," Soil Dynamics and Earthquake Engineering, vol. 144, Article ID 106709, 2021.

[20] L. C. Li, W. B. Wu, H. Liu, and B. Lehane, "DEM analysis of the plugging effect of open-ended pile during the installation process," Ocean Engineering, vol. 220, Article ID 108375, 2021.

[21] M. A. Biot, "Theory of propagation of elastic waves in a fluidsaturated porous solid. I. Low-Frequency range," The Journal of the Acoustical Society of America, vol. 28, no. 2, pp. 168-178, 1956.

[22] M. A. Biot, "Mechanics of deformation and acoustic propagation in porous media," Journal of Applied Physics, vol. 33, no. 4, pp. 1482-1498, 1962.

[23] K. Sahebkar and M. Eskandari-Ghadi, "Time-harmonic response of saturated porous transversely isotropic half-space under surface tractions," Journal of Hydrology, vol. 537, pp. 61-73, 2016.

[24] Z. Zhang and E. Pan, "Time-harmonic response of transversely isotropic and layered poroelastic half-spaces under general buried loads," Applied Mathematical Modelling, vol. 80, pp. 426-453, 2020.

[25] Y. Cai, G. Chen, C. Xu, and D. Wu, "Torsional response of pile embedded in a poroelastic medium," Soil Dynamics and Earthquake Engineering, vol. 26, no. 12, pp. 1143-1148, 2006.

[26] K. Wang, Z. Zhang, C. J. Leo, and K. Xie, "Dynamic torsional response of an end bearing pile in saturated poroelastic medium," Computers and Geotechnics, vol. 35, no. 3, pp. $450-458,2008$

[27] G. Wang, W. Ge, X. Pan, and Z. Wang, "Torsional vibrations of single piles embedded in saturated medium," Computers and Geotechnics, vol. 35, no. 1, pp. 11-21, 2008.

[28] Z. Li, Y. Gao, and K. Wang, "Torsional vibration of an end bearing pile embedded in radially inhomogeneous saturated soil," Computers and Geotechnics, vol. 108, pp. 117-130, 2019.

[29] Z. Zhang and E. Pan, "Vertical vibration of a rigid circular disc embedded in a transversely isotropic and layered poroelastic half-space," Engineering Analysis with Boundary Elements, vol. 118, pp. 84-95, 2020.

[30] W. B. Wu, X. L. Xu, H. Liu, C. L. Fang, B. Dou, and R. Z. Liang, "Vertical vibration characteristics of a variable impedance pile embedded in layered soil," Mathematical Problems in Engineering, vol. 2017, Article ID 1794950, 11 pages, 2017.
[31] D. J. Liu, Y. Z. Liu, and J. Y. Wang, "Theoretical study on shear wave applied in low strain dynamic testing of piles," Chinese Journal of Geotechnical Engineering, vol. 25, no. 3, pp. 283287, 2003, in Chinese.

[32] O. C. Zienkiewicz, C. T. Chang, and P. Bettess, "Drained, undrained, consolidating and dynamic behaviour assumptions in soils," Géotechnique, vol. 30, no. 4, pp. 385-395, 1980. 